\title{
Politiques des objets et objets politiques
}

Les adductions d'eau villageoises en Afrique de l'Ouest

Fabrice Gangneron

\section{(2) OpenEdition}

\section{Journals}

Édition électronique

URL : http://journals.openedition.org/anthropodev/600

DOI : 10.4000/anthropodev.600

ISSN : 2553-1719

Éditeur

APAD - Association pour l'anthropologie du changement social et du développement

\section{Édition imprimée}

Date de publication : 1 décembre 2017

Pagination : 127-149

ISBN : 979-10-93476-05-6

ISSN : 2276-2019

\section{Référence électronique}

Fabrice Gangneron, «Politiques des objets et objets politiques », Anthropologie \& développement [En ligne], 46-47 | 2017, mis en ligne le 01 juin 2018, consulté le 19 avril 2019. URL : http:// journals.openedition.org/anthropodev/600 ; DOI : 10.4000/anthropodev.600

\section{(c) (7)}

La revue Anthropologie \& développement est mise à disposition selon les termes de la Licence Creative Commons Attribution 4.0 International. 


\title{
Politiques des objets et objets politiques
}

\section{Les adductions d'eau villageoises en Afrique de l'Ouest}

\author{
Fabrice Gangneron
}

L'objectif de cet article est de discuter des dispositifs sociotechniques que sont les adductions d'eau en milieu semi-urbain en Afrique de l'Ouest, d'interroger trois aspects qui tous peuvent être qualifiés de politiques. (1) Leurs "matérialités organisées" façonnées de main d'homme, projettent des scénarios qui exercent un pouvoir d'action sur la nature des usages et les modes de gestion (l'outil contraint). (2) Leurs inscriptions concrètes dans les univers sociotechniques de leurs usages montrent qu'ils "s'accordent" avec les humains qui en ont la charge, qui les interprètent (l'outil "coopère »). (3) Enfin, les discours des bailleurs de fonds, des acteurs politiques et des opérateurs de développement sur les dispositifs confinent au mythe technologique d'un développementisme unilinéaire (l'outil est instrumentalisé par le discours).

The aim of this paper is to discuss three political dimensions of a sociotechnical device, namely water supply systems, in West African semi-urban areas. Due to their man-made "organized materialities", they embed assumptions on how they are to be used, and thus acquire the capacity to constrain the nature of use and the way they are to be managed (the technical tool constrains). However, the way they are embedded in sociotechnical practices through their use also shows that water supply systems "agree" with the people in charge of them, who "interpret" them (the technical tool cooperates). Finally, the discourses of donor agencies or development actors about water supply (re)produce technological myths that draw on a "linear developmentist" approach in which the technical tool is instrumentalised. 


\section{Introduction}

Cet article propose une réflexion qui s'appuie sur des expériences, ancrées essentiellement au Bénin et au Burkina Faso ainsi qu'au Niger, au Mali et au Sénégal ${ }^{1}$. Il est donc issu d'un travail de terrain, lequel a nourri une réflexion sur ce qui est de l'ordre du politique. Les politiques publiques de ces pays d'Afrique de l'Ouest n'apparaissent qu'en filigrane dans ce texte parce qu'elles font l'objet d'assez nombreuses publications dans le domaine de l'eau (Hounmenou, 2006; Diop et Dia, 2011; Jaglin et Belbeoc'h, 2010 ; Bonnassieux et Gangneron, 2011 ; Baron et Maillefert, 2011 ; Valette et $a l ., 2017^{2}$ ). Un élément structurant la réflexion de cet article considère qu'il s'agit de pays qui sont "sous régime d'aide" (Lavigne Delville, 2010), donc largement contraints par les bailleurs, même si leurs gouvernements conservent des marges de manœuvre. Ils ont notamment amorcé un processus de désengagement de l'État par la promulgation de lois de décentralisation. Ils ont procédé, au même moment, à des reformulations des politiques de distribution d'eau potable en milieu rural et semi-urbain (de 2000 à 10000 habitants pour le semiurbain). L'article propose d'analyser les dimensions politiques de dispositifs technologiques, ici les puits, les pompes et surtout les adductions qui sont

${ }^{1}$ Ces travaux se sont déroulés essentiellement dans le cadre d'un programme financé par I'INSU "Analyse multidisciplinaire de la mousson africaine » (AMMA, 2009-2012) et dans le cadre de l'ANR «Action publique éclatée. Production et institutionnalisation de l'action publique dans les secteurs de l'eau potable et du foncier. Bénin, Burkina Faso, Niger » (APPI, 2011-2014).

${ }^{2}$ L'article en collaboration avec H. Valette et A. Bonnassieux (Valette et al., 2017) paru dans le précédent numéro de la revue Anthropologie \& développement fait écho à celui-ci. Leurs contenus se complètent mais les approches sont sensiblement différentes. Ils se complètent dans la mesure où ils montrent l'un et l'autre la tendance aux implantations d'adductions d'eau villageoises (AEV) dans une perspective de construction de petits marchés semi-urbains de l'eau. Le précédent article inscrit sa réflexion dans le " référentiel marchand » que nous déclinons selon quelques-uns de ses grands principes : la décentralisation, la délégation de service, la tarification et la participation à l'échelle nationale (en l'occurrence le Bénin) et locale (les deux communes de Djougou et de Kpomassè que I'on retrouve aussi dans cet article). À la différence du précédent, celui-ci s'attache à interroger les dispositifs techniques, en l'occurrence les AEV conçues, non pas comme de simples outils au service des politiques de l'eau mais comme étant eux-mêmes des dispositifs politiques. 
au centre de ce texte, alors que ces dispositifs sont généralement classés et interprétés (par les opérateurs de développement, ainsi que par nombre de chercheurs du domaine) comme de simples transmetteurs neutres de progrès.

\section{Contextualisation, problématisation}

Depuis la DIEPA (Décennie internationale de l'eau potable et de l'assainissement, 1981-1990), puis la " Déclaration du millénaire " (Nations Unies, 2000) qui avait pour objectif de réduire de moitié le nombre de personnes n'ayant pas accès à l'eau potable à l'horizon 2015, les pays d'Afrique de l'Ouest ont bénéficié de nombreux programmes de couverture en eau potable tant en zone urbaine qu'en milieu rural. Cet article propose une réflexion essentiellement en zone semi-urbaine où les choix techniques et les modes de gestion ont connu une évolution notable, ainsi que des transitions sociotechniques (remplacement des puits par des PMH, puis des $\mathrm{PMH}$ par des $\mathrm{AEV}^{3}$ ) propres à modifier le rapport des usagers et des gestionnaires à l'eau. Dans les années 1980, l'action publique (États, ONG, coopération) faisait encore porter ses efforts sur les réhabilitations des puits et les fonçages de nouveaux puits busés. On leur a ensuite préféré les $\mathrm{PMH}$ puis, dans les années 2000 , les programmes se sont progressivement orientés vers l'implantation d'AEV. Les couvertures en eau potable devaient ainsi être sensiblement améliorées, le passage du puits à la PMH assurant une meilleure qualité de l'eau puisée et celui de la $\mathrm{PMH}$ à I'AEV une plus grande couverture villageoise de sorte à progresser sur la voie de la démocratie hydrique. Les arguments en faveur de cette modernisation empruntent largement à la rhétorique sanitaire et de

\footnotetext{
${ }^{3}$ Selon les pays ou les auteurs la terminologie change légèrement, les $\mathrm{PMH}$ (pompes à motricité humaine) sont aussi appelées FPM (forages équipés de pompes à motricité humaine), tandis que les AEV (adductions d'eau villageoises) peuvent être désignées sous le vocable d'AEP (adductions d'eau potable sommaires ou simplifiées). Les transitions techniques (ou sociotechniques) désignent les orientations des politiques et des bailleurs de fonds vers des dispositifs techniques plus sophistiqués, pour autant les dispositifs plus anciens (puits et pompes en particulier) sont encore utilisés, simplement ils ne sont plus - en milieu semi-urbain - privilégiés par les politiques de développement.
} 
progrès propre au champ du développement, voire au "développementisme ".

La culture développementiste telle que l'indiquent certains auteurs (Faugère, 2000; Lavigne Delville et Thiéba, 2015) conduit à des raisonnements et à des discours techniques (augmenter le taux de couverture, promouvoir la gestion professionnalisée...) et moraux (accéder au "progrès", culpabiliser les usagers pour leur recours aux eaux insalubres...). Elle est un paradigme unilinéaire et téléologique, "une idéologie du temps linéaire » (Gras, 2003, cité par Guchet, 2005). Les pays du Nord, montrant " la voie de la modernité », exercent leur magistère par des programmes et des politiques qui ne laissent guère de place à des processus alternatifs ou pluriels. Ignacy Sachs (1981) avait tenté d'ouvrir le débat d'un "écodéveloppement ", appuyant sa réflexion, entre autres choses, sur les potentiels des cultures locales, propres à réinterpréter le développement à la lumière de la pluralité des histoires culturelles et politiques des pays du Sud. La notion de développement durable ${ }^{4}$, plus consensuelle et plus homogénéisante lui fut préférée; elle s'appuie largement sur les transferts de technologie et l'ancrage aux modèles économiques du Nord. Cette notion laisse peu de place au buissonnement, à la pluralité des voies techniques et des modèles de gestion, au profit d'un processus de remplacement afin d'entrer dans " La Modernité » conçue au Nord, comme unique et prédéfinie.

Les critiques adressées au développementisme dans le secteur de l'eau portent pour l'essentiel sur la dépolitisation de la question hydrique. Elles montrent notamment l'émergence d'une économie marchande de l'eau et interrogent les limites et/ou les ambiguïtés de la libéralisation du secteur ainsi que de ses outils : partenariat public-privé (PPP), décentralisation... Ce sont les voies politiques choisies par les bailleurs et les États qui sont alors visées et les capacités du marché à permettre aux populations les plus démunies d'accéder au service de l'eau. Sont notamment critiqués la

\footnotetext{
${ }^{4}$ Le développement durable n'est certes pas une alternative à l'écodéveloppement (Sachs luimême avait défendu la notion de développement durable au Sommet de Rio de 1992), mais l'écodéveloppement est un développement plus endogène qui ouvre à une pluralité de voies possibles.
} 
(re)qualification de la ressource en termes économiques (Calvo-Mendieta et al., 2010), l'alignement des États africains sur les modèles occidentaux (Darbon, 2003), les adhésions de fait au référentiel marchand (Baron et Maillefert, 2011) et les échecs (ou du moins les bilans mitigés) des voies marchandes (Jaglin, 2005 ; Bonnassieux et Gangneron, 2011). Dans ces analyses critiques, c'est " le politique " qui est questionné, les institutions, le poids des bailleurs, la formation et la circulation des élites qui reproduisent et diffusent les "modèles dominants". Le politique est ainsi conçu comme un jeu d' " humains " qui s'accordent ou se disputent et qui, pour atteindre des "objectifs", utilisent des objets, des machines, des dispositifs techniques, simples instruments passifs dans la main des hommes. C'est la main que l'on questionne, l'objet n'étant entendu que comme une extension mécanique pour augmenter son pouvoir d'action sur le monde.

Pour l'eau villageoise, les opérateurs et experts en développement s'interrogent généralement sur la manière de prolonger l'action publique par des objets ou des dispositifs techniques; ce faisant ils font d'eux des entités a-sociales, a-politiques. La démarche de cet article consiste à interroger ces dispositifs techniques comme le fait la sociologie des sciences et des techniques (Latour, 2001), afin de traiter "les choses comme des faits sociaux ". Les objets sont d'ordinaire refoulés dans un mode sans signifiant, comme le montre Simondon (1958), seule leur qualité d'instrument de progrès est communément interprétée. II est pourtant possible de questionner les objets eux-mêmes, dans leur " intériorité » (Akrich, 1987 et 1989), dans leur matière façonnée de main d'homme, et de considérer qu'ils inscrivent en eux des usages et des modalités d'accès à l'eau, qu'ils distribuent, assignent des rôles aux humains et cadrent les relations objets/humains. Pour montrer leur pouvoir d'action sur les hommes, la sociologie de la traduction fait des dispositifs techniques des actants (Akrich, Callon et Latour, 2006). Simondon parle d'individus. Ils sont des entités " agissantes" qui formulent des scénarios d'inscription dans leurs univers de destination. Pour chaque objet ou dispositif, "le concepteur avance un certain nombre d'hypothèses sur les éléments qui composent le monde dans lequel l'objet est destiné à s'insérer » (Akrich, $2010: 208)$, lesquelles orientent, cadrent usages et modalités d'usage. En conséquence, la pluralité des technologies 
imprime une pluralité de cadres sociotechniques, de telle sorte que les usagers n'accèdent pas à proprement parler à l'eau mais à des ressources plurielles médiées par des dispositifs.

Simondon (1958) s'intéresse d'avantage à l'" intelligence " des objets techniques qu'aux acteurs les produisant, ils ne sont que des "hommes", des "humains " sans distinction. II n'identifie pas de producteurs, d'usagers, ni les processus itératifs de coproduction qui mêlent ou requalifient usages et conceptions. Pour aller plus loin dans l'analyse, il devient donc nécessaire de discuter plus précisément des acteurs. La " construction sociale des technologies" (social construction of technology ou SCOT) apporte des clarifications. L'idée phare consiste à ouvrir la boîte noire de la coproduction, de mieux identifier et "socialiser " les acteurs, les faisant passer d' "humains" abstraits à des groupes sociaux identifiables. Wiebe et al. (2002), Pinch (2008), et d'autres, proposent de prendre de la distance avec la dichotomie producteurs/usagers pour développer le concept de "relevant social group " plus à même selon eux d'illustrer les processus de production des objets techniques. Les auteurs relèvent que la bicyclette, la musique électronique, la lampe, la radio, le téléphone, ne sont pas à proprement parler des productions de concepteurs, séparés d'usagers qui seraient de simples utilisateurs selon les modalités canoniques d'un unique mode d'emploi. Les concepteurs et les usagers sont redéfinis pour illustrer les interactions qui président à la coconstruction, ou même pour montrer que certains sont à la fois les uns et les autres. L'histoire de la naissance du synthétiseur (Pinch, 2008) illustre bien cette approche avec les figures de musiciens/ingénieurs d'avant-garde des années 1960 à la fois inscrits dans les savoirs techniques nouveaux (transistors, oscillateurs, filtres, amplificateurs...) et dans des courants musicaux contestataires. La micro-électronique industrielle a permis la miniaturisation (donc la transportabilité) à des coûts raisonnables. Elle a aussi permis des sons totalement nouveaux que la culture underground new-yorkaise a testés, triés, modifiés, aboutissant par itération à transformer un objet expérimental en synthétiseur.

Dans le paradigme de coproduction de la SCOT, les relevant groups développent une " intelligence distribuée " propre à configurer la technologie, c'est-à-dire l'objet physique et l'usage. Ces processus d'innovation supposent un monde social en proximité géographique et culturelle, des 
" passeurs " et donc des passages (des lieux et des moments) via des institutions pouvant lier conceptions et usages. Les dispositifs de type FPM et AEV d'Afrique de l'Ouest sont difficilement assimilables aux illustrations proposées par la SCOT à moins de procéder à un déplacement de la problématique.

Darbon montre que la construction des États ouest-africains procède de "l'administration projetée " (Darbon, 2003), fondée sur des stratégies de transferts de modèles politiques et de technologies pensés au Nord par des producteurs de normes conçues comme des "référentiels universels". Ces administrations déploient un ensemble de procédures et de dispositifs concrets (Lascoumes et Le Galès, 2005), dont les " technologies projetées » que sont les AEV. Les "designers", pensent les AEV depuis des positions distanciées du monde de destination. Les passeurs qui assureraient une connectivité Nord/Sud, voire Sud/Sud, de coproducteurs sont fort rares. Une remarquable exception est toutefois à relever au Zimbabwe (De Laet et $\mathrm{Mol}, 2000$ ) où une pompe à main (la " bush pump "), pensée avec les utilisateurs, est l'aboutissement en plusieurs générations de prototypes améliorés, à un outil extrêmement rustique, peu sujet aux pannes et facile à réparer sans recourir à des professionnels. Aucun processus de ce type ne semble s'être produit en Afrique de l'Ouest.

Aussi, postulant une discontinuité, il est ici proposé de traiter des dispositifs techniques en deux parties distinctes. La première les présente ex situ, en exposant leurs propriétés intrinsèques et leurs pouvoirs d'action sur le monde de destination. Une seconde partie propose de mettre à l'épreuve ces propriétés par les pratiques concrètes: les politiques publiques et les arrangements opérés par les acteurs impliqués (notables locaux, élus, techniciens, gestionnaires, employés, fontainières, usagers). In fine le fonctionnement concret (règles locales, institutions) est issu à la fois de la physicalité du dispositif de médiation (puits, $\mathrm{PMH}, \mathrm{AEV}$ ) et de son inscription sociotechnique dans son contexte d'usage où chaque acteur, depuis sa position d'usager, de gestionnaire, de réparateur..., exerce une certaine réflexivité interprétative lui permettant de (re)configurer ses relations avec les dispositifs. 


\section{Trois cadres sociotechniques}

Cette partie propose une description détaillée de la matérialité des dispositifs techniques, de leur intériorité et, ainsi, de leurs "intentions » qui procèdent d'une " mise en ordre du monde " (Berten, 1999). L'idée est d'interroger le sens commun, de déconstruire la conception instrumentale de la technique. Ce sens commun ne constitue pas un point de vue erroné - la technique reste un moyen pour arriver à des fins - mais partiel. Elle est cela, mais elle est aussi autre chose que rend nécessaire une entreprise de dévoilement.

L'ordre de présentation des dispositifs ci-dessous (puits, PMH, AEV) reflète la chronologie des politiques de modernisation par paliers symboliquement inscrits dans la téléologique du progrès technique. Cette partie n'ambitionne pas d'exposer les scripts d'usage et de gestion des trois dispositifs techniques du point de vue des concepteurs, mais de restituer à partir des dispositifs techniques eux-mêmes les systèmes relationnels qu'ils projettent dans les espaces sociaux concrets de leurs usages.

L'eau médiée par le puits est une ressource multi-usages, pour la boisson, la cuisine, la lessive, pour l'abreuvement des animaux d'élevage et même pour l'arrosage de petits jardins. Qu'il soit de facture locale (c'est-àdire qu'il mobilise l'organisation, les savoirs et les matériaux locaux nécessaires à sa fabrication), ou issu de techniques et matériaux industriels comme les buses en béton, l'exhaure ne nécessite qu'une corde, parfois des fourches en bois ou des poulies, un seau ou une outre. Les matériaux de base pour l'exhaure sont facilement mobilisables dans l'environnement proche, bois pour les fourches ou les poulies, fibres végétales ou tissus (de sac de céréales par exemple) pour la fabrication de cordes. Les coûts de ces accessoires sont nuls ou modestes. L'énergie pour l'exhaure est fournie par l'usager ou un animal domestique. Le puits ne nécessite pas d'entretien pour son usage (sauf lorsqu'il n'est pas busé, en particulier en zone sédimentaire). II n'a pas de panne ${ }^{5}$, il ne requiert pas de gestion ni de paiement. Si son usage est souvent régulé, c'est pour des raisons externes

${ }^{5}$ II peut s'effondrer, dans ce cas nous ne sommes plus dans le cadre des scripts de sa gestion ordinaire. 
à l'objet lui-même. Ces raisons ont un lien, non pas avec la matérialité du puits, mais avec son environnement social et spatial. II n'est en effet pas rare qu'un notable, qu'un groupe, qu'un village, s'approprie la ressource ou simplement fasse valoir des droits de gestion et organise son accès (paiements imposés à des éleveurs allochtones, établissements de priorités ou de tranches horaires d'approvisionnement en saison sèche).

La PMH constitue une première rupture dans le processus développementiste. Avec son bras de levier en métal, ses tuyaux en plastique, ses joints, clapets, ressorts et son cylindre, elle est sujette aux pannes, elle suppose un suivi de maintenance et des réparations. Les matériaux qui constituent le dispositif, de facture industrielle, ne permettent pas de réparations sans recourir à l'argent (Gangneron et al., 2010). Le coût peut évidemment être pris en charge par la puissance publique ou les usagers, mais le passage au paiement est incontournable ${ }^{6}$. La PMH ne conduit pas pour autant à la " marchandisation » de la ressource, elle ne constitue pas un enjeu économique pour quelque gestionnaire que ce soit sauf peut-être pour un marché de la réparation. Le scénario que produit la $\mathrm{PMH}$ est celui d'une petite autonomie locale, de volumes modestes, d'un service de proximité pour une communauté d'usagers réduite. Les pays destinataires traduisent ces dispositions par une desserte pour 400 personnes au Mali, 300 au Burkina Faso. Sans énergie thermique ni électrique, sans compteur volumétrique, la $\mathrm{PMH}$ n'inscrit pas en elle le paiement au volume, elle le rend toutefois possible par l'usage de bassines calibrées. Le paiement est d'ailleurs encouragé par les politiques publiques (Direction nationale de

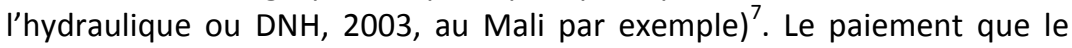
dispositif implique, quel que soit le payeur (usager, communauté, puissance publique) est conciliable avec un système forfaitaire.

\footnotetext{
${ }^{6}$ Les paiements peuvent prendre bien des aspects, ils peuvent être préventifs avec par exemple des cotisations forfaitaires d'usagers. Ils peuvent être curatifs (lorsque la panne survient), le village ou des notables du village se cotisent pour le paiement du dépannage. Le paiement peut être totalement contourné, alors, lorsque la panne survient, la pompe reste hors d'usage.

${ }^{7}$ Les arguments avancés sont la « responsabilisation » des usagers et leur participation aux coûts de fonctionnement.
} 
L'AEV est l'archétype de ce que Guchet (2005) appelle "l'essence technique " dans ses trois fonctions : extraire, stocker et transporter ; trois fonctions génériques auxquelles on peut ajouter deux fonctions spécifiques, traiter et distribuer. Elle inclut de nouveaux éléments techniques. Pour l'extraction de l'eau, elle a un ou plusieurs forages, l'exhaure est assurée par un moteur thermique ou électrique relié au réseau ou à un système photovoltaïque. Le château d'eau stocke en hauteur quelques mètres cubes ou dizaines de mètres cubes d'eau qui $y$ résident suffisamment longtemps pour permettre son assainissement. Le remplissage peut être automatique ou manuel sous le contrôle d'un opérateur. Les quantités pompées sont contrôlées par un compteur général. L'eau est répartie dans un système de tuyauterie rayonnant depuis le centre, le réservoir, jusqu'à la périphérie, les usagers. Des vannes divisionnaires permettent d'isoler des segments du réseau de ce système arborescent sans fermer l'ensemble pour anticiper la maintenance ou les pannes locales. Les extrémités sont composées de deux types de distribution, l'un en extérieur sous forme de bornes fontaines (BF) collectives, l'autre à l'intérieur des habitats sous forme de branchements privés (BP). Chaque BF dispose de robinets et d'un compteur. II en va de même avec les BP mais là, les usages sont individuels (une famille ou une institution). Le dispositif projette la mise en paiement. Par la présence des compteurs ( $\mathrm{BP}, \mathrm{BF}$ et compteur général), le système planifie des calculs de rendement eau distribuée/eau pompée, donc des fuites et des coûts d'exploitation (dépenses énergétiques pour l'exhaure).

Ce dispositif technique fait émerger des fonctions professionnelles (plombiers, mécaniciens, électriciens, gestionnaires), donc des accès payants. La dispersion dans l'espace villageois des BF et des BP lui confère un potentiel de distribution à quelques milliers d'usagers. Le dispositif ne devient pas nécessairement marchand mais il inscrit en lui les éléments qui permettent ce passage. L'AEV ouvre à cette possibilité par cette nécessaire professionnalisation et sa technicité, par les dépenses énergétiques pour l'exhaure et le contrôle du débit, par un possible horizon de consommations massives dont une des clés est le déploiement des branchements individuels. Par rapport aux dispositifs plus rustiques, elle opère un partiel 
" désencastrement " (ou " disembeddedness " ${ }^{8}$, selon Polanyi, 1983). II peut s'illustrer sommairement ainsi, le dispositif de médiation que constitue la PMH définit des relations humains/ressource essentiellement en termes de rapports sociaux. Les fonctions de distribution se satisfont d'une simple présence sans opération technique à mener, les fontainières étant en quelque sorte au service de la petite communauté des usagers, tandis que la gestion à proprement parler est sans enjeu économique mais constitue au contraire un fort enjeu social pour les usagers. Les PMH ne requièrent pas d'institutions spécifiques pour fonctionner même si elles sont livrées avec un "mode d'emploi " (voir par exemple, DNH, Mali, 2004) contraignant les usagers/gestionnaires à une organisation formelle. Au contraire, par les tâches techniques et de gestion, par ses capacités de distribution, l'AEV contraint la gestion à des fonctions spécifiques qui tendent soit à extraire le dispositif technique des institutions locales (fonctions assurées par des professionnels non villageois) soit à intégrer des nouvelles fonctions aux institutions villageoises (formations techniques de notables anciens gestionnaires bénévoles). Elle est en capacité de (co)produire des rapports économiques et marchands par l'émergence d'employés, donc du salariat, d'une gestion entrepreneuriale visant à dégager des profits d'exploitation.

\footnotetext{
${ }^{8}$ Encastrement (ou enchâssement) est une traduction de l'anglais " embeddedness ", concept qui met en lumière une propriété des économies pré ou non industrielles. Dans ces dernières, les sphères économiques et sociales étant enchevêtrées, l'économie est gérée à des fins sociales ou politiques et les institutions sont non spécialisées (à la fois économiques et sociales). Pour rester très proche du terrain et sans discuter plus avant ou même rentrer dans de très longs débats qui ont cours en sociologie économique, les encastrements locaux peuvent être décrits ainsi : les relations économiques médiées par les dispositifs techniques peuvent être vues comme encastrées lorsqu'aucune institution locale spécialisée n'en a la charge (pas ou peu de recours aux institutions économiques que sont les banques, pas de professionnels de gestion...). Ces fonctions sont prises en charge par des institutions coutumières (notables, chefferies, collectifs masculins) qui participent de tout ce qui constitue la vie du village, quelles que soient les apparences d'un formalisme administratif qu'elles peuvent prendre (comité ou association de gestion). Lorsque la charge de la gestion est sous la responsabilité d'un entrepreneur, que le système recourt à des professionnels et un dépôt de banque, lorsque la ressource s'inscrit dans le marché, il y a désencastrement, au moins relatif, au moins en processus. La discussion sur la question n'est toutefois jamais close, par exemple l'entrepreneur peut lui-même être un notable du village, être issu d'un lignage qui compte dans les rapports sociaux...
} 


\section{La vie des AEV}

\section{Les pratiques : l'AEV coopère avec les humains}

Les politiques publiques ont semble-t-il tâtonné avant de fixer des formes de gestion qui tendent à se stabiliser, s'institutionnaliser. Les premières $\mathrm{AEV}$ sont en gestion dite communautaire alors que, depuis la Conférence de Dublin de 1992, se diffuse le "référentiel marchand" (Baron et Maillefert, 2011) pour l'eau potable en milieu semi-urbain. Le référentiel s'opérationnalise, certes par tâtonnements, mais au travers d'un processus commun aux pays d'Afrique de l'Ouest associant invariablement décentralisation, délégation, contractualisation, participation et tarification (République du Bénin, 2008 ; Valette et al., 2017). La gestion déléguée à des entrepreneurs devient progressivement la norme. Pourtant elle révèle de très nombreuses difficultés techniques et économiques, d'absentéisme des fermiers, de résistances au paiement, de détournements d'argent, de difficultés de gestion des fonds collectés..., qui conduisent à de longs arrêts, voire à des abandons d'AEV. Malgré ces difficultés, le Bénin en particulier se conforme aux "préconisations" internationales de délégation. Si le secteur n'est actuellement pas encore totalement encadré, ni techniquement opérationnel, deux exemples illustrent le processus de délégation et de construction de marchés semi-urbains s'appuyant sur les AEV, l'un dans la commune de Djougou (département de l'Atacora-Donga), l'autre dans celle de Kpomassè (département de l'Atlantique).

Hors agglomération, Djougou dispose de six AEV dont la gestion est toujours soumise à un certain nombre d'aléas, mentionnés plus haut, montrant que l'environnement humain et sociotechnique est peu préparé aux politiques de délégation. La gestion se décline ainsi : un contrat tripartite entre la commune, un entrepreneur et l'Association des consommateurs d'eau potable (ACEP). L'ACEP, héritière des organisations communautaires de gestion, n'exerce plus qu'une fonction de veille, réduite à une ou deux réunions annuelles, sans pouvoir décisionnel. Sur le site de chaque AEV est affecté un employé (de l'entrepreneur concessionnaire) dont la fonction, brièvement décrite, est la collecte des paiements aux BF, le relevé des compteurs de BF et de BP, le contrôle du niveau d'eau, l'appoint de gasoil pour le moteur diesel, la réparation des 
petites pannes, et enfin la transmission de toute information majeure à son employeur (panne importante...). Cet employé est un opérateur aux compétences basiques, il est forcément lettré et il a les bases techniques que requiert le dispositif, ainsi que le schéma contractuel. La distribution de l'eau aux BF est assurée par une fontainière qui délivre l'eau en bassines calibrées et fait payer les usagers.

La vie des AEV n'est pourtant pas totalement conforme au schéma institué et le formalisme contractuel est largement revisité par les pratiques locales. L'argent de l'eau rentre mal, les fontainières ne maîtrisent pas toujours les sommes remises, les usagers ont des stratégies pour éviter le paiement. Les consommations sont souvent faibles. L'entrepreneur, absentéiste, n'exerce pas de suivi et ne confie pas de fonds de roulement à son employé, lequel est irrégulièrement rémunéré. Lorsqu'une panne complexe ou onéreuse survient, l'employeur doit donner son accord pour utiliser les fonds disponibles afin de s'adresser à un professionnel du dépannage qui se fournira chez un commerçant spécialisé... Sauf que cette chaîne d'exécution n'existe pas, l'employeur est généralement injoignable, les caisses sont peu fournies, il n'y a ni professionnel, ni magasin à proximité. Ce sont les arrangements locaux, la maîtrise des incertitudes par des employés débrouillards, qui sont la clé du fonctionnement. Quand la panne arrive, ils utilisent les voies typiquement " encastrées ». Pour les fonds, ce sont les notables qui participent (et font savoir leur participation de sorte à asseoir leur notabilité) ou l'ensemble du village qui est sollicité. S'il faut mobiliser de la main-d'œuvre, ils font appel à des jeunes pour creuser, maçonner, charrier..., qu'ils rémunèrent sur les recettes des BF. Il leur arrive de faire eux-mêmes les achats de pièces et matériaux sans recourir à l'entrepreneur.

A contrario, pour deux des AEV de la commune de Kpomassè, le rôle de l'employé se rapproche du schéma institué et la division du travail y est plus avancée. L'employeur est plus présent, les procédures de gestion, d'entretien et de dépannage sont, sinon totalement conformes au cahier des charges, du moins elles s'en rapprochent. Le point de vue d'un employé qui avait connu l'époque précédente (en gestion communautaire) témoigne d'un appauvrissement de sa fonction. II ne fait plus les relevés qui sont confiés à un autre agent, lequel recourt à l'informatique, il fait les tournées de maintenance et les petites réparations. II assure le 
remplissage et l'assainissement du château d'eau, il transmet toute information utile à son employeur qui se charge des décisions. Les fonds de roulement sont remis en banque, sous le contrôle de l'entrepreneur. La proximité de la capitale économique (Cotonou est à $70 \mathrm{~km}$ ) permet d'accéder aux magasins de pièces de rechange.

La fonction de fontainière connaît elle aussi une sensible transformation. Issue de la communauté villageoise, la fontainière de PMH est généralement une femme âgée et/ou veuve et/ou handicapée qui n'a pas la force physique d'exercer une activité rémunératrice ou de cultiver les champs. Elle régule les accès à la pompe et procède au paiement s'il est au volume. Elle reçoit un pourcentage sur les ventes, l'équivalent d'un ou deux repas quotidiens. Son amplitude horaire peut aller du lever au coucher du soleil. Le service fonctionne grâce à sa disponibilité. Les premières $A E V$ installées dans les deux communes ont fonctionné un temps sur ce mode avec les mêmes profils. Mais à Kpomassè, l'émergence de la professionnalisation de l'ensemble du dispositif sociotechnique a fait naître un enjeu économique qui transforme la fonction. Elle devient peu à peu un travail là où les consommations croissent et où la rigueur comptable s'installe. Les fontainières doivent alors maîtriser les calculs de base (volumes, conversions en argent, lecture du compteur...) et commencent à tirer quelques profits économiques de la fonction (Bonnassieux et Gangneron, 2015). De fait, sur les AEV les plus fréquentées, les fontainières âgées laissent peu à peu la place à des femmes plus jeunes pour lesquelles la BF devient source de revenu, directement grâce aux consommations et indirectement par les petits commerces de proximité qu'elles entretiennent au pied de la BF. Elles y vendent des produits de première nécessité tels que riz, farine de manioc et sucre achetés sur les bénéfices des consommations d'eau. Certaines ont même de véritables boutiques.

Les exemples béninois témoignent d'une pluralité d'arrangements sociotechniques. Ils montrent, aussi, l'accord d'une double prescription, celle du dispositif technique (I'AEV) et celle des orientations politiques dans leur projet de faire émerger des marchés semi-urbains de l'eau (Valette et al., 2015). D'autres pays de la région ont fait montre de plus d'originalité politique, exerçant ainsi leur pouvoir d'action, même à la marge. Au Sénégal, la gestion est confiée soit à des entrepreneurs, soit aux 
Associations des usagers de forages (ASUFOR). Les associations mettent I'AEV en gérance sous des formes contractuelles qui relèvent de la cogestion (les ASUFOR reçoivent tout ou partie des recettes d'exploitation, elles sont en position de cogestionnaires). De même au Niger, les gestions d'AEV sont relativement hétérogènes avec des essais d'affermage qui cohabitent avec des gestions communautaires. Au sud-ouest du Burkina Faso, dans la région de Bobo Dioulasso, une Association pour le développement des adductions d'eau (ADAE) a mis en pratique une gestion originale, sans contester le marché (Baron et Bonnassieux, 2013) et compatible avec les scénarios des dispositifs techniques. Elle soutient les gestionnaires qui sont constitués en association, dans une démarche de gestion partagée ; elle a contribué à fédérer le suivi technique des AEV et à mettre en place un système de péréquation entre centres déficitaires et excédentaires, permettant ainsi de maintenir la continuité du service et un prix unique de l'eau sur l'ensemble des AEV. Ces exemples montrent des écarts entre les cadres programmatiques des politiques publiques de délégation de services et les parcours processuels (Mosse et al., 1998) conduisant à des arrangements non prévus pouvant s'institutionnaliser : le Burkina a reconnu la gestion délégataire de l'ADAE.

Dans tous les cas, les bilans sont complexes, mais les AEV qui fournissent l'eau avec régularité sont celles dont les scénarios techniques ont trouvé leurs acteurs: entrepreneurs ou associations, employés débrouillards et/ou aux fortes compétences techniques, fontainières... Du seul point de vue de la continuité du service, la gestion déléguée n'est pas une condition nécessaire au bon fonctionnement du dispositif sociotechnique. Bien que les contraintes exercées par les dispositifs techniques produisent normes, règles et scénarios spécifiques, ils composent, s'accordent avec les choix politiques, constituant une alchimie dynamique entre déterminisme technologique et (non pas contre) constructivisme social (Vincent et Forest, 2010).

\section{Le mythe : l'AEV est instrumentalisée}

Les potentiels techniques des AEV pour couvrir les besoins en eau potable du plus grand nombre apparaissent comme une promesse de progrès sanitaire et démocratique. À première vue leurs succès n'en seraient que la conséquence logique. Leurs avantages sur les PMH sont-ils pour autant 
aussi nets et systématiques en tous lieux et en tous contextes sociaux ? Le succès des $A E V$ se comprend plus aisément à travers une analyse du récit sur le progrès technique dont elles sont l'actuelle incarnation.

Les récits ne sont pas une production des dispositifs techniques ou de leurs pratiques, ils en sont des interprétations, ils projettent du sens, ils produisent un système signifiant (ici comme vecteurs de progrès, outils salvateurs). Barthes (1957) montre que le sens, produit par interprétation, se révèle dans la relation des deux pôles sémiologiques, le " signifiant » et le "signifié ". Le premier terme se réfère à la matérialité, aux objets concrets, qui renvoient au second, au domaine de l'idéel, aux représentations. Le signifié n'est pas un simple miroir de l'objet, il le " rend présent " par l'interprétation qui en est faite. II est au-delà du vrai ou du faux. Le récit producteur de mythe n'évacue pas l'objet, il le met en perspective, il le "purifie » et il le "fonde en nature ». De plus, pour Barthes, le mythe dépolitise, alors que même les objets les plus modestes contiennent des "traces politiques ". Le mythe indique ce qui est bon à son auto-entretien en masquant (volontairement ou non) certaines propriétés. Bouchard (2013) s'accorde à cette idée ajoutant que le mythe est porteur de croyances (ici d'égalité, de progrès, d'accès à l'eau pour tous...). Il en propose cette définition :

"[...] stratégiquement produit et utilisé, le mythe social est une représentation collective hybride, bénéfique ou nuisible, baignant dans le sacré, commandée par l'émotion plus que par la raison, et porteuse de sens, de valeurs et d'idéaux façonnés dans un environnement social et historique donné » (Bouchard, 2013 : 68).

Le récit mythifiant est celui de la modernité technologique s'incarnant dans I'AEV qui libère de l'insécurité hydrique par la disponibilité permanente d'une ressource de bonne qualité sanitaire, pour le plus grand nombre. Ce récit est relativement homogène chez les bailleurs, dans les ministères, au sein de la coopération, et plus localement dans les services décentralisés, les communes et les ONG qui, tous, sont portés par un élan de glorification de la technique.

Mais qu'il s'agisse de stratégies claires ou non, les discours font des impasses sur les effets des choix sociotechniques (c'est-à-dire du duo $\mathrm{AEV} /$ gestion délégataire). Les résistances constatées à consommer l'eau 
des AEV apparaissent généralement comme des faits d'arrière-garde appelés à disparaître. Pour que l'AEV soit acceptée de tous, il est indispensable que le récit se diffuse jusqu'aux usagers, qu'il faut bien convaincre d'abandonner les ressources alternatives. II faut communiquer pour parvenir à l'acceptabilité sociale afin que les usagers recourent le plus possible à l'AEV, il faut maximiser les avantages des choix politiques et minimiser les problèmes posés. Pour cela, la transmission du récit est souvent déléguée à des ONG d'intermédiation sociale. Elles ont généralement la charge de dispenser des formations techniques et financières (auprès des gestionnaires, des exploitants) et de diffuser le récit jusqu'aux populations afin d'en obtenir l'acceptation à payer. Certaines AEV de Kpomassè ont d'ailleurs vu leurs consommations d'eau augmenter en partie grâce au travail de ces ONG. L'adhésion au dispositif reste toutefois à géométrie variable, plus nette dans la commune de Kpomassè que dans celle de Djougou où les AEV sont en gestion délégataire (qui ont donc mis le paiement au cœur du système sociotechnique), et où l'usage des AEV n'est pas toujours socialement accepté ni économiquement supportable par tous. Le paiement a émergé avec la $\mathrm{PMH}$, mais à des niveaux restant modestes avec des possibilités de forfait et de gratuité étendue. La ressource médiée par l'AEV est immanquablement plus onéreuse mais, lorsque l'AEV est gérée par un système bénévole, le prix global de l'eau est contenu dans le coût de l'énergie et de l'entretien du dispositif. Avec l'affermage, ce sont les professionnels et l'entrepreneur qu'il s'agit en plus de rémunérer. Au total, l'eau qui n'excède pas 100 à $150 \mathrm{FCFA} / \mathrm{m}^{3}$ avec les $\mathrm{PMH}$, atteint actuellement 450 à $600 \mathrm{FCFA} / \mathrm{m}^{3}$ sous gestion délégataire ${ }^{9}$ dans tous les pays d'Afrique de l'Ouest. De ce fait, les usagers ont des stratégies d'évitement, certains utilisent les ressources alternatives gratuites ou moins chères tant qu'elles sont disponibles, tandis que les plus démunis n'utilisent

\footnotetext{
${ }^{9}$ Notons qu'il s'agit de coûts visibles, ceux des contrats d'affermage. Dans la pratique, le coût de l'eau aux AEV peut être encore plus élevé comme dans les cas de revente (donc avec des petits bénéfices) à partir des $\mathrm{BP}$, ou quand l'eau est acheminée par un porteur d'eau depuis les BF jusque dans des quartiers non couverts, ou encore dans les cas singuliers de fontainières qui ont des stratégies pour augmenter leurs marges en s'arrangeant sur les volumes des bassines, plus petits que ne le prévoit le contrat, au prix indiqué par le contrat.
} 
pratiquement pas les AEV. Ajoutons que la gestion par un fermier produit entre autre effet de désencastrement celui d'éloigner les usagers (plus précisément les notables locaux) de la maîtrise du dispositif, ceux-ci devenant de simples clients de l'eau. Les arrangements locaux tels qu'ils ont été restitués au Nord-Bénin, vont sans doute devenir marginaux par la volonté publique de délégation.

Le duo AEV/gestion privée, générant augmentation des prix et phénomène partiel de désencastrement, ne produit pas une meilleure couverture partout et pour tous, il est somme toute assez loin d'une réelle démocratie hydrique. La littérature grise et les institutions nationales font état de taux de couverture qui prennent peu en compte les pratiques réelles des populations (saisonnalité des usages, stratégies d'acteurs, mécanismes de protection sanitaire...) : une $\mathrm{PMH}$ équivaudrait à 300400 personnes, une BF à 250-300 ou encore un BP à 30 personnes $^{10}$. Sans doute faudrait-il tenter de revisiter le taux de couverture et ne pas s'en satisfaire s'il atteint les $100 \%$ visés, pour produire un " taux d'usage effectif " qui signifierait d'analyser les pratiques à toute saison et pour toutes les populations.

Le mythe est entretenu par la coopération internationale, les États, les passeurs de discours, les opérateurs de développement (en particulier des ONG du secteur de l'eau) et les acteurs privés. Il est au service d'un projet politique qui mêle d'authentiques préoccupations sanitaires à des visées de constitution de petits marchés locaux de l'eau. Dans leur rhétorique, ces acteurs reprennent à leur compte la "professionnalisation du secteur ", terminologie consensuelle pour éluder un mouvement de privatisation de la gestion porté par l'idée d'efficacité économique du secteur privé, qui, selon Benjaminsen et Svarstad (2009), appartient au "petit nombre de discours" construits autour de ce que Baron et Maillefert (2011) appellent le référentiel marchand. Le marché, même

${ }^{10} \mathrm{D}$ 'après un entretien anonyme avec un cadre béninois du ministère de l'Énergie, des Recherches pétrolières et minières, de l'Eau et du Développement des énergies renouvelables, le ministère considère qu'un branchement privé alimente 7,6 personnes par foyer et 3,9 foyers (en considérant qu'il y a revente de l'eau), ce qui fait 29,64 personnes par branchement. 
amendé d'un contrôle public, constitue l'horizon de la " bonne gestion ». La rhétorique s'appuie pour cela sur les échecs des gestions communautaires comme justifications d'un passage à une gestion sinon totalement privée du moins sous forme de PPP.

\section{Conclusion}

Les débats qui interrogent la technique sont déjà anciens et relativement tranchés entre deux positions, celle du déterminisme technologique face à celle du constructivisme social (Vincent et Forest, 2010). Ceux qui ont questionné le déterminisme ont mis en lumière les capacités de la technique à interpréter et à transformer le monde humain, certains la considérant comme un outil, un media politique d'aliénation, d'asservissement (Ellul, 1977; Heidegger, 1958). De même, Simondon (1958) rappelle que la technique a le pouvoir de "modeler la civilisation " mais, pour lui, l'aliénation n'est pas une fatalité. Si les objets et dispositifs techniques incluent de l'humain, donc des "intentions", des scénarios d'usage, ce sont les méconnaissances de ces intentions qui produisent l'aliénation, alors que la construction de savoirs qu'on peut requalifier de sociotechniques permet d'en faire des " alliés ", c'est-à-dire d'en explorer toutes leurs potentialités. Ces savoirs doivent donc pouvoir se construire en interrogeant l'objet lui-même, en explorant ses scénarios, les contraintes qu'il exerce sur les systèmes d'acteurs (mise en paiement, dépendance énergétique, choix ou non d'une pluralité de dispositifs...), travail amont de démythification, sans doute même de désenchantement. Akrich (1987 et 1989) et Olivier de Sardan (2001) montrent une certaine plasticité des dispositifs qui sont interprétés dans le contexte de leurs usages (communes, entrepreneurs, employés, fontainières, usagers), ils rappellent en cela au travers des systèmes sociotechniques qu'on ne peut évacuer l'approche constructiviste. Les AEV " coopèrent " et les schémas formalisés de gestion en PPP sont d'évidence revisités, au moins dans certains cas, en particulier dans les situations décrites plus haut comme encastrées, aux multiples arrangements locaux. Cet encastrement peut d'ailleurs être vu comme une condition de la pérennité, sinon du mythe, du moins du service. 
Toutefois la plasticité du dispositif AEV a des limites, le paiement reste incontournable, des compétences spécifiques lui sont attachées, l'individualisation de l'usage (grâce aux BP) est inscrite dans le scénario technique et l'usage reste essentiellement domestique. Le duo AEV/choix politiques de gestion déléguée servi par le récit du progrès a conduit sur un " sentier de la dépendance " (Guchet, 2005 ; Jaglin, 2012) technique et politique. Les AEV dominent le paysage semi-urbain où elles ont remplacé (ou tendent à le faire) les dispositifs plus rustiques (puits et $\mathrm{PMH}$ en particulier), les bailleurs et les politiques nationales ne se réfèrent plus guère qu'à elles et à leur gestion déléguée. Pourtant, les bilans jusqu'à aujourd'hui des gestions " professionnelles » n'ont pas toujours produit les résultats escomptés (les dispositifs connaissent de très nombreuses pannes, longues à réparer, les problèmes comptables sont légion...), alors que les discours les présentent comme l'unique voie. D'autres voies, une pluralité de voies, permettraient de répondre à certains des soucis que rencontrent les usagers, notamment celui du coût de l'eau, de l'importance des ressources alternatives et de la pluralité des usages. Elles appellent à envisager plusieurs modes de gestion pour un même dispositif (typiquement pour les AEV), ainsi qu'une pluralité de dispositifs (puits couverts et $\mathrm{PMH}$ ). Finalement, la question n'est plus de savoir si la technique libère ou si elle aliène, mais de comprendre que les choix sociotechniques du moment déplacent les contraintes et qu'une pluralité de possibles permettrait de choisir entre ces contraintes.

\section{Bibliographie}

AKRICH M., 1987, "Comment décrire les objets techniques? ", Technique et culture, $9: 49-64$.

AKRICH M., 1989, "La construction d'un système sociotechnique. Esquisse pour une anthropologie des techniques", Anthropologie et société, 2(13): 31-54.

AKRICH M, CALLON M., LATOUR B., 2006, Sociologie de la traduction: textes fondateurs, Paris, Presse des Mines. 
BARON C., BONNASSIEUX A., 2013, " Gouvernance hybride, participation et accès à l'eau potable. Le cas des associations d'usagers de l'eau (AUE) au Burkina Faso ", Annales de Géographie, 693 : 525-548.

BARON C., MAILLEFERT M., 2011, « Une lecture institutionnelle de la gouvernance de l'eau potable: des terrains d'Afrique de l'Ouest francophone aux faits stylisés ", Régions \& Cohésion, 3(1) : 7-33.

BARTHES R., 1957, Mythologies, Paris, Seuil.

BENJAMINSEN T.A., SVARSTAD H., 2009, "Qu'est-ce que la "political ecology" ? ", Natures Sciences Société, 1(17) : 3-11.

BERTEN A., 1999, « Dispositifs, médiation, créativité : petite généalogie », Hermès, $25: 35-47$.

BONNASSIEUX A., GANGNERON F., 2011, « Des mini-réseaux d'eau potable : entre enjeux politiques et arrangements locaux. Le cas de la commune de Djougou au Bénin ", Mondes en Développement, 155 : 77-92.

BONNASSIEUX A., GANGNERON F., 2015, "Les fontainières, des actrices subalternes et pourtant incontournables du service de l'eau en Afrique de I'Ouest (Bénin, Burkina Faso et Niger) ", colloque CARE, Université Lyon 3, 7-10 septembre 2015.

BOUCHARD G., 2013, "Pour une nouvelle sociologie des mythes sociaux ", Revue européenne des sciences sociales, 1(51) : 95-120.

CALVO-MENDIETA I., PETIT O., VIVIEN F.D., 2010, «Entre bien marchand et patrimoine commun, l'eau au centre des débats de l'économie de l'environnement ", in SCHNEIER-MADANES G. (Dir), L'eau mondialisée La gouvernance en question, Paris, Éditions La Découverte, Collection Recherches : 61-74.

DARBON D., 2003, "Réformer ou renforcer les administrations projetées des Afriques?", Revue française d'administration publique, 105-106: 138152.

DE LAET M., MOL A., 2000, "The Zimbabwe bush pump: mechanics of a fluid technology ", Social studies of science, 2(30) : 225-263.

DIOP M., DIA A.H., 2011, "Réformes des services d'eau en milieu rural africain : enjeux et limites du montage institutionnel de gestion. Une étude de cas au Sénégal ", Mondes en développement, 155 : 37-68. 
DiRECTION NATIONALE DE L'HYDRAUliQue (DNH, MALI), 2003, «Principes directeurs pour la gestion des pompes à motricité humaine », $6 \mathrm{p}$.

DIRECTION NATIONALE DE L'HYDRAULIQUE (DNH, MALI), 2004, " Modèle de convention de délégation de gestion des pompes à motricité humaine ", $5 \mathrm{p}$.

ELLUL J., 1977, Le système technicien, Paris, Calmann-Lévy.

FAUGÈRE E., 2000, "Regards sur la culture développementiste : représentation et effets non intentionnels ", Document scientifique, $n^{\circ} 20$, GRED, Paris, $28 \mathrm{p}$.

GANGNERON F., BECERRA S., DIA A.H., 2010, « Des pompes et des hommes. État des lieux des pompes à motricité humaine d'une commune du Gourma malien », Autrepart, 55 : 39-56.

GRAS A., 2003, La fragilité de la puissance. Se libérer de l'emprise technologique, Fayard.

GUCHET X., 2005, Les sens de l'évolution technique, Éditions Léo Scheer.

HEIDEGGER M, 1958, "La question technique », in Essais et conférences, Paris, Gallimard, collection « Tel », $n^{\circ} 52: 9-48$.

HOUNMENOU B.G., 2006, "Gouvernance de l'eau potable et dynamiques locales en zone rurale au Bénin ", Développement durable et territoire, dossier $n^{\circ} 6$, les territoires de l'eau.

JAGLIN S., 2005, " La participation au service du néolibéralisme ? Les usagers dans les services d'eau en Afrique subsaharienne ", in BACQUÉ M.H., REY U., SYNTOMER Y., Gestion de proximité et démocratie participative, une perspective comparative, La Découverte : 271-291.

JAGLIN S., 2012, "Services en réseau et villes africaines : I'universalité par d'autres voies? ", L'espace géographique, 1(41) : 51-67.

JAGLIN S., BELBEOC'H A., 2010, "Services d'eau et décentralisation en Afrique de l'Ouest: réflexions à partir de petites villes du Bénin et du Mali ", in SCHNEIDER-MADAMES G., L'eau mondialisée, la gouvernance en question, Paris, La découverte : 199-218.

LASCOUMES P., LE GALÈS P., 2005, Gouverner par les instruments, Paris, Presses de Sciences po.

LATOUR B., 2001, L'espoir de pandore. Pour une version réaliste de l'activité scientifique, Paris, La découverte. 
LAVIGNE DELVILLE P. 2010, "La réforme foncière rurale au Bénin : émergence et mise en question d'une politique instituante dans un pays sous régime d'aide ", Revue française de science politique, 60(3) : 467-491.

LAVIGNE DELVILLE P., THIÉBA D., 2015, « Débat public et production des politiques publiques au Burkina Faso. La politique nationale de sécurisation foncière ", Participations, $11:$ 213-236.

MOSSE D., FARRINGTON J. et REW A. (éds), 1998, Development as process. Concepts and methods for working with complexity, London, Routledge/ODI.

NATIONS UNIES, 2000, "Déclaration du millénaire ", assemblée générale des Nations Unies, 6-8 septembre 2000, New York.

OLIVIER DE SARDAN J.P. et DAGOBI A.E., 2001, " La gestion des pompes dans le département de Tillabery ", LASDEL, Études et travaux, $n^{\circ} 4$.

PINCH T., 2008, "Technology and institutions : in a material world ", Theory and society, 5(37) : 461-483.

POLANYI K., 1983, La grande transformation. Aux origines politiques et économiques de notre temps, Paris, Gallimard, $440 \mathrm{p}$.

RÉPUBLIQUE DU BÉNIN, 2008, « Politique nationale de l'eau », 51 p.

SACHS Y., 1981, Initiation à l'écodéveloppement, Toulouse, Privat.

SIMONDON G., 1958, Du mode d'existence des objets techniques, Paris, Aubier.

VALETTE H., BARON C., ENTEN F., LAVIGNE DELVILLE P., TSITSIKALIS A., 2015, « Une action publique éclatée? Production et institutionnalisation de l'action publique dans les secteurs de l'eau potable et du foncier (APPI), Burkina Faso, Niger, Bénin ", Actes colloque, 2-4 décembre 2014, Toulouse, Gret.

VALETTE H., GANGNERON F., BONNASSIEUX A., 2017, " L'intégration et la mise en œuvre des principes marchands dans le secteur de l'eau en milieu rural et semi-urbain béninois ", Anthropologie \& développement, 45 : 113-142.

VINCENT P., FOREST J., 2010, "Réformes des services urbains en Inde ", Revue Tiers Monde, 203 : 81-102.

WIEBE E., BILKER E., PINCH T., 2002, « SCOT, Answers questions: a reply to Nick Clayton », Technology and culture, 2(43) : 361-370. 


\section{Fabrice Gangneron}

\section{iviviviv}

Fabrice Gangneron est socio-géographe

laboratoire géosciences environnement Toulouse (GET), université Paul Sabatier, IRD, CNRS

E-mail : fabrice.gangneron@get.obs-mip.fr 